\title{
Autoimmune Hepatitis Refractory to Treatment Due to Underlying Grave's Disease
}

\author{
Rahul Sawhney ${ }^{1}$, Sadhna Dhingra ${ }^{2}$, Gagan K. Sood ${ }^{3}$ \\ 1. Internal Medicine, Baylor University Medical Center, Dallas, USA 2. Anatomic Pathology, Baylor \\ College of Medicine, Houston, USA 3. Abdominal Transplantation, Baylor College of Medicine, Houston, \\ USA
}

$\square$ Corresponding author: Rahul Sawhney, rahulsaw07@gmail.com

Disclosures can be found in Additional Information at the end of the article

\section{Abstract}

A 24-year-old Hispanic woman presented to our facility with a two-week history of abdominal pain, nausea, vomiting, diarrhea, jaundice, and scleral icterus. Initial laboratory workup revealed elevated transaminases, direct hyperbilirubinemia, and positive anti-smooth muscle antibody. Liver biopsy confirmed the diagnosis of autoimmune hepatitis and our patient was started on oral prednisone therapy. Her liver enzymes initially began to normalize but then spontaneously started up-trending. She was subsequently readmitted to the hospital for further management, at which time she also complained of palpitations, heat intolerance, and sweating. Laboratory workup revealed hyperthyroidism secondary to Grave’s disease. Our patient was not a candidate for methimazole or propylthiouracil treatment due to her hepatic dysfunction, so she was started on hydrocortisone due to its secondary effect of decreased conversion of thyroxine to triiodothyronine. She achieved biochemical remission of her autoimmune hepatitis on this regimen and was transitioned back to oral prednisone therapy. Her liver enzymes normalized once she underwent radioactive iodine ablation of her thyroid. This clinical course suggests that autoimmune hepatitis with concurrent Grave's disease may be refractory to treatment until the underlying hyperthyroid state is corrected.

Received 04/19/2019

Review began 04/24/2019

Review ended 04/30/2019

Published 05/04/2019

CC Copyright 2019

Sawhney et al. This is an open access article distributed under the terms of the Creative Commons Attribution License CC-BY 3.0., which permits unrestricted use, distribution, and reproduction in any medium, provided the original author and source are credited.
Categories: Endocrinology/Diabetes/Metabolism, Internal Medicine, Gastroenterology Keywords: hepatitis, autoimmune hepatitis, liver disease, grave's disease, hyperthyroidism, mmi, aih, propylthiouracil, methimazole, ptu

\section{Introduction}

Autoimmune hepatitis (AIH) is a chronic inflammatory condition characterized by hepatocellular inflammation secondary to autoantibody-induced lymphocytic proliferation [1]. The presentation of AIH is varied, ranging from patients who are asymptomatic to those who present with acute liver failure [2]. AIH is commonly known to be associated with other autoimmune diseases [3]. We present a case of AIH with associated Grave's disease in which the underlying hyperthyroidism seemingly contributed to progressive liver dysfunction.

\section{Case Presentation}

A 24-year-old Hispanic woman with a past medical history of post-partum pre-eclampsia presented to the emergency department with a two-week history of abdominal pain, nausea, vomiting, diarrhea, jaundice, and scleral icterus. On a review of systems, she denied recent travel, sick contacts, hepatotoxic medications or supplements, or parenteral exposure risks. She 


\section{Cureus}

reported a distant history of tattoos placed with clean needles and had undergone a cesarean section three months prior, with the delivery of a healthy baby boy. Aside from obesity, she denied other risk factors for liver disease.

Her physical exam was unremarkable except for obesity, jaundice, and scleral icterus. The patient had no exophthalmos, goiter, or tachycardia. Initial laboratory workup revealed elevated liver function tests, including aspartate transaminase (AST) 1,041 IU/L (8-48 IU/L), alanine transaminase (ALT) $809 \mathrm{IU} / \mathrm{L}$ (7-55 IU/L), total bilirubin $11.5 \mathrm{mg} / \mathrm{dL}(0.1-1.2 \mathrm{mg} / \mathrm{dL})$, direct bilirubin $8.7 \mathrm{mg} / \mathrm{dL}$ ( $<0.3 \mathrm{mg} / \mathrm{dL}$ ), and prothrombin time 15.1 seconds (9.5-13.8 seconds). Anti-smooth muscle antibody (ASMA) was positive and immunoglobulin G (IgG) was elevated at 2,288 $\mathrm{mg} / \mathrm{dL}$ (767-1,590 mg/dL). Anti-nuclear/anti-mitochondrial antibodies and hepatitis viral serologies were negative. Iron/copper studies were within normal limits. Thyroid studies were not performed at this time, as she did not exhibit any clinical evidence of thyroid dysfunction.

Subsequently, an ultrasound-guided core needle liver biopsy was performed, which showed interface hepatitis with extensive portal lymphocytic infiltration, mild to moderate (Grade 2-3) periportal and lobular inflammation, portal fibrosis, and early (Stage 1-2) portal-to-portal septa (Figure 1), consistent with a diagnosis of autoimmune hepatitis.
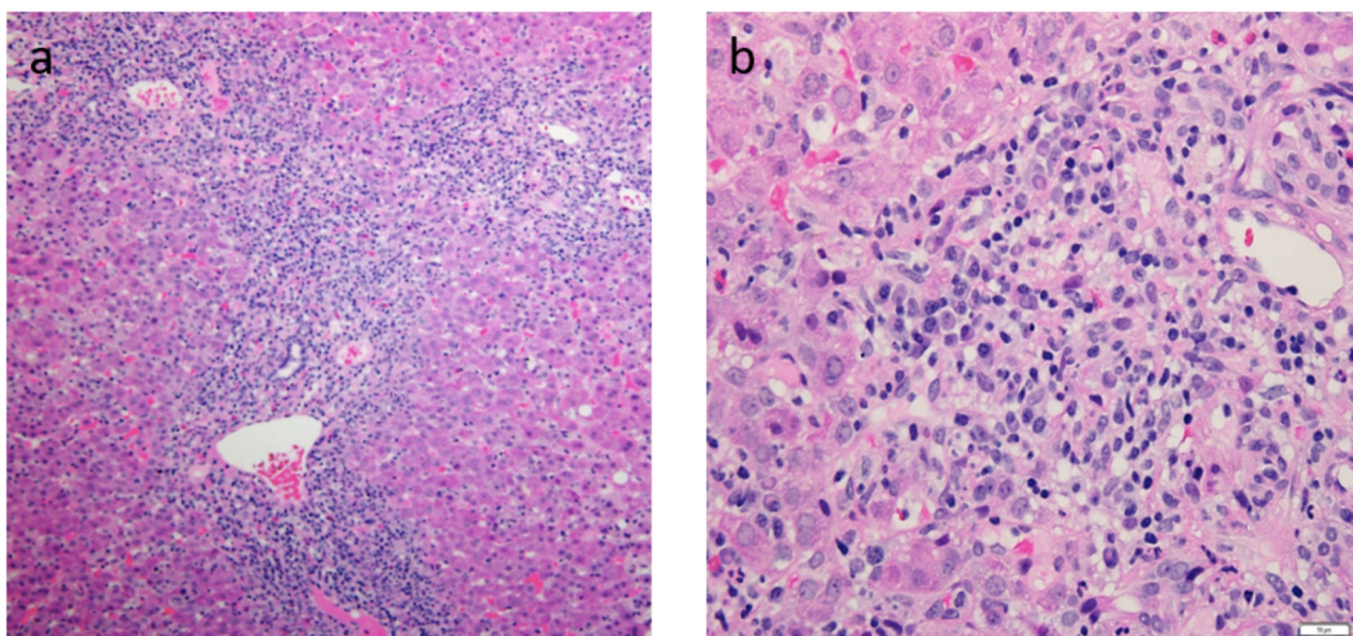

\section{FIGURE 1: Liver biopsy slides of findings consistent with autoimmune hepatitis.}

(a) Portal expansion by chronic lymphoplasmacytic inflammation with moderate to severe interface hepatitis. (b) Inflammatory infiltrate includes clusters of plasma cells admixed with lymphocytes and few eosinophils.

The probability of our patient having AIH was calculated using a standardized scoring system. Based on the presence of ASMA, IgG elevation >1.1 times the upper limit of normal, histology compatible with a diagnosis of AIH, and negative viral serologies, the patient's score was 6 as per the simplified AIH scoring system. This was indicative of a probable diagnosis of AIH [2,45]. Human leukocyte antigen (HLA) allele was not tested.

Treatment was started with high-dose prednisone to be tapered from $60 \mathrm{mg}$ to $40 \mathrm{mg}$ after two weeks. Azathioprine $50 \mathrm{mg}$ daily was added after three weeks. Initially, her transaminases and total bilirubin trended downward, but these values sharply increased at the six-week mark (Figure 2). She also complained of palpitations, sweating, and heat intolerance, which 
prompted readmission for further evaluation. Additional workup revealed underlying hyperthyroidism with thyroid-stimulating hormone (TSH) $<0.01 \mu \mathrm{IU} / \mathrm{mL}(0.4-5.5 \mu \mathrm{IU} / \mathrm{mL})$, free triiodothyronine (T3) $11.5 \mathrm{pg} / \mathrm{mL}$ (2.8-4.4 pg/mL), and free thyroxine (T4) $39.5 \mathrm{ng} / \mathrm{dL}$ (0.9-1.7 $\mathrm{ng} / \mathrm{dL})$. Grave's disease was confirmed with a radioactive iodine uptake of $44 \%$ (8-25\%) and positive thyroid-stimulating immunoglobulin.

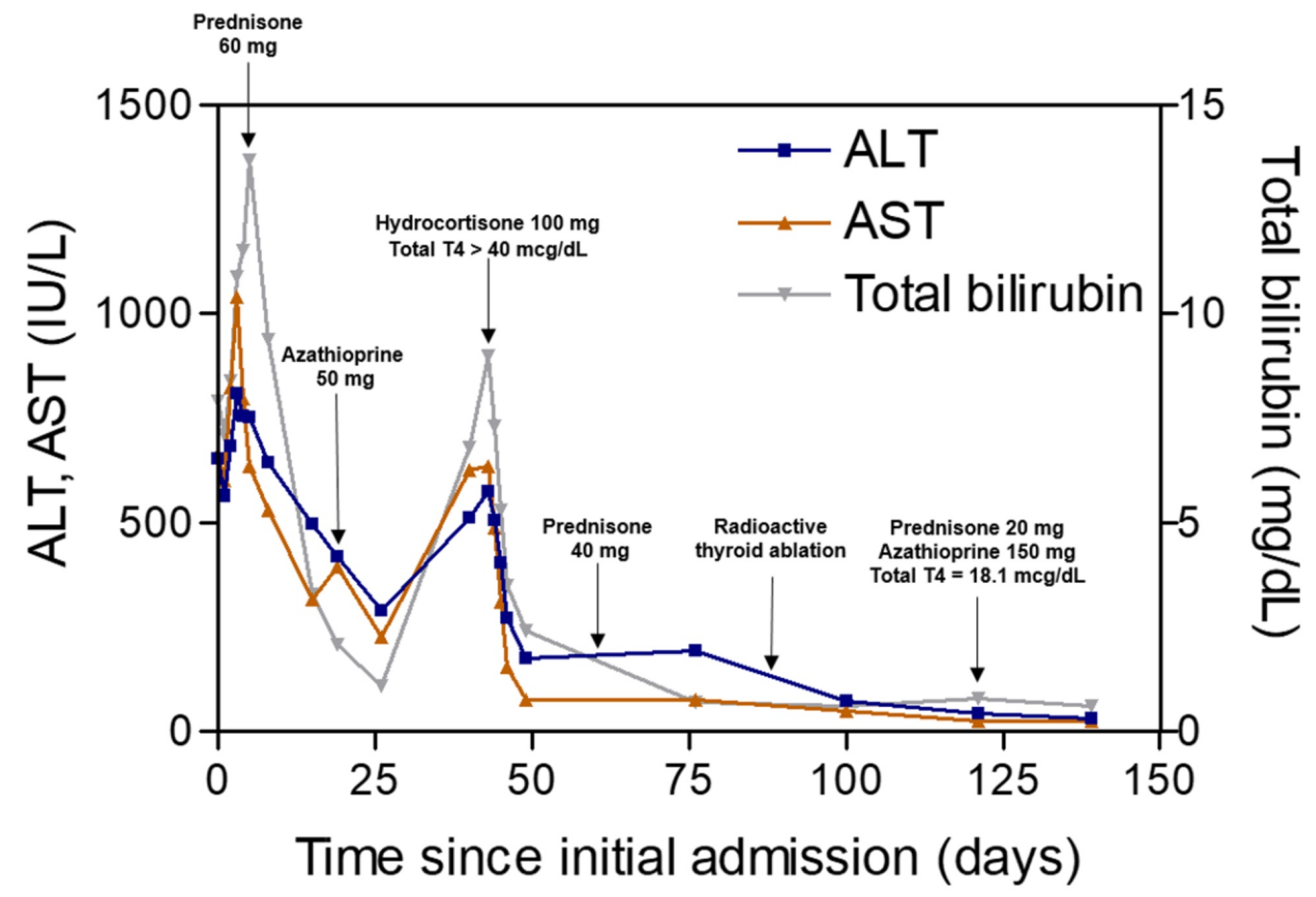

FIGURE 2: Patient's clinical course since time of admission.

ALT: alanine aminotransferase; AST: aspartate aminotransferase; T4: thyroxine.

Given her hepatic function, the patient was not a candidate for standard therapy with methimazole or propylthiouracil. She was instead started on high-dose hydrocortisone, which provided an added benefit of decreased peripheral conversion of T4 to T3 [6]. Her hyperthyroid symptoms improved while AST, ALT, and total bilirubin decreased by nearly $75 \%$ within one week. This represented the first time in three months that the patient's transaminases dropped below $200 \mathrm{IU} / \mathrm{L}$. Subsequently, she underwent radioactive thyroid ablation and began levothyroxine supplementation. Her AST, ALT, and total bilirubin also normalized - signifying the biochemical remission of AIH - once she received the ablation. Afterward, the patient remained stable on standard immunosuppressive therapy, with $20 \mathrm{mg}$ prednisone and $150 \mathrm{mg}$ azathioprine daily.

\section{Discussion}

Our patient was diagnosed with autoimmune hepatitis based on positive autoimmune serology (anti-smooth muscle antibody), elevated IgG, and compatible histopathological findings. Standard immunosuppressive therapy with prednisone and azathioprine yielded a suboptimal response. An additional diagnosis of Grave's disease was made based on clinical findings, low TSH, elevated free T3 and T4, positive thyroid stimulating immunoglobulin, and increased radioactive iodine uptake. 
disease, and rheumatoid arthritis, have been described in nearly $40 \%$ of patients with AIH. However, the concurrence of additional autoimmune diseases does not usually alter the clinical course or severity of AIH [3,7].

Initial treatment for AIH includes high-dose prednisone daily, to which our patient had a suboptimal response [2]. Upon her diagnosis of Grave's disease, our patient was started on intravenous hydrocortisone, as methimazole and propylthiouracil were contraindicated given her liver function. This regimen led to markedly improved control of the patient's AIH. Hydrocortisone, along with suppressing the immune system, prevents the conversion of T4 to T3 and can ameliorate a hyperthyroid state [6].

Hyperthyroidism has been reported to be associated with hepatic function abnormalities through several mechanisms of liver dysfunction. These include liver abnormalities due to hyperthyroidism alone, liver damage related to heart failure and hyperthyroidism, and concomitant liver disease in the setting of hyperthyroidism [2]. Our patient had no signs of heart failure and the persistent elevation of her liver aminotransferases despite immunosuppressive therapy was attributed to the associated hyperthyroid state.

Biochemical remission was achieved with adequate control of the underlying hyperthyroidism and maintained with low-dose prednisone and azathioprine. Our patient achieved a euthyroid state after radioactive ablation of her thyroid gland and subsequent oral levothyroxine supplementation.

\section{Conclusions}

Our case highlights that an underlying hyperthyroid state can contribute to hepatic dysfunction and may cause suboptimal responsivity of AIH to standard immunosuppressive therapy. The recognition and management of associated hyperthyroidism can aid in achieving an optimal biochemical response and avoiding unnecessary incremental increases in the immunosuppressive regimen. Based on our findings, we recommend that patients with AIH refractory to therapy be evaluated for possible thyroid disease.

\section{Additional Information \\ Disclosures}

Human subjects: Consent was obtained by all participants in this study. Baylor College of Medicine Institutional Review Board issued approval N/A. From IRB handbook: "Case reports or case series describing interesting observations on three or fewer patients do not meet the definition of research as a systematic investigation designed to contribute to generalizable knowledge. Therefore the IRB does not review or approve such reports. Case series including more than three patients will be considered research and will require IRB review and approval prior to the conduct of the research. Even though a case series of three or fewer patients does not constitute research, it is still subject to HIPAA rules. Investigators therefore may be required to de-identify all data disclosed (as defined by HIPAA) or to obtain patient authorization to disclose private health information.". Conflicts of interest: In compliance with the ICMJE uniform disclosure form, all authors declare the following: Payment/services info: All authors have declared that no financial support was received from any organization for the submitted work. Financial relationships: All authors have declared that they have no financial relationships at present or within the previous three years with any organizations that might have an interest in the submitted work. Other relationships: All authors have declared that there are no other relationships or activities that could appear to have influenced the submitted work. 


\section{Cureus}

\section{References}

1. Webb GJ, Hirschfield GM, Krawitt EL, Gershwin ME: Cellular and molecular mechanisms of autoimmune hepatitis. Annu Rev Pathol. 2018, 13:247-292. 10.1146/annurev-pathol-020117043534

2. Manns MP, Czaja AJ, Gorham JD, Krawitt EL, Mieli-Vergani G, Vergani D, Vierling JM: Diagnosis and management of autoimmune hepatitis . Hepatology. 2010, 51:2193-2213. 10.1002/hep.23584

3. Teufel A, Weinmann A, Kahaly GJ, et al.: Concurrent autoimmune diseases in patients with autoimmune hepatitis. J Clin Gastroenterol. 2010, 44:208-213. 10.1097/MCG.0b013e3181c74e0d

4. Hennes EM, Zeniya M, Czaja AJ, et al.: Simplified criteria for the diagnosis of autoimmune hepatitis. Hepatology. 2008, 48:169-176. 10.1002/hep.22322

5. Alvarez F, Berg PA, Bianchi FB, et al.: International Autoimmune Hepatitis Group report: review of criteria for diagnosis of autoimmune hepatitis. J Hepatol. 1999, 31:929-938. 10.1016/S0168-8278(99)80297-9

6. Carroll R, Matfin G: Endocrine and metabolic emergencies: thyroid storm. Ther Adv Endocrinol Metab. 2010, 1:139-145. 10.1177/2042018810382481

7. Khemichian S, Fong TL: Hepatic dysfunction in hyperthyroidism. Gastroenterol Hepatol (N Y). 2011, 7:337-339. 